\title{
SOBRE LA (IN)COMPETENCIA DE LAS INSTITUCIONES EUROPEAS PARA REFORMAR LOS SISTEMAS PÚBLICOS DE PENSIONES NACIONALES A LA LUZ DEL DERECHO DE LA UE Y DE LOS TRATADOS INTERNACIONALES
}

\author{
ABOUT THE (IN)COMPETENCE OF THE EUROPEAN \\ INSTITUTIONS TO REFORM THE PUBLIC SYSTEMS \\ OF NATIONAL PENSIONS IN THE LIGHT OF THE EU \\ LAW AND THE INTERNATIONAL TREATIES
}

\author{
Cristina SÁnchez-Rodas Navarro \\ Catedrática de Derecho del Trabajo y Seguridad Social \\ Universidad de Sevilla* \\ ORCID ID: 0000-0001-9780-7860
}

Recibido: 15.01.2018 / Aceptado: 06.02.2018

DOI: https://doi.org/10.20318/cdt.2018.4127

\begin{abstract}
Resumen: En España, tras la crisis mundial económica y financiera de 2008, se han llevado a cabo importantes reformas legislativas a fin de controlar el déficit público y cumplir así no sólo con las disposiciones emanadas del Derecho de la Unión Europea sino también con los Tratados internacionales ratificados por nuestro país.

Por su cuantía, las pensiones contributivas de jubilación constituyen la partida más importante de los Presupuestos Generales del Estado.

Existe una generalizada creencia de que las restrictivas reformas en materia de pensiones españolas que se vienen promulgando en los últimos años son inevitables al venir impuestas por disposiciones emanadas de la Unión Europea y/o por Tratados internacionales.

El objeto de este artículo es, por una parte, demostrar que la competencia para legislar en materia de Seguridad Social es, y sigue siendo, competencia exclusiva del Estado. Y, por otro lado, verificar cómo las reformas en materia de pensiones obedecen a iniciativas legislativas nacionales, en modo alguno impuestas por el Derecho de la UE o el Derecho internacional.
\end{abstract}

Palabras clave: Unión Europea, Seguridad Social, pensiones contributivas de jubilación.

Abstract: In Spain, after the worldwide economic and financial crisis of 2008, important legislative reforms have been carried out in order to control the public deficit and therefore will be able to comply not only with the provisions emanating from the European Union Law but also with the International Treaties ratified by our country.

Due to their amount, contributory old-age pensions are the most important item in the General State Budget.

There is a widespread belief that the restrictive reforms on Spanish pensions that have been enacted in recent years were inevitable because they were imposed by provisions emanating from the European Union and /or international treaties.

*Esta publicación se enmarca dentro de los trabajos de investigación desarrollados en el Proyecto I+D “La Seguridad Social Internacional y Comunitaria" (DER 2017-83040-C4-3-R) financiado por el MINECO. 
The purpose of this article is, on the one hand, to demonstrate that the competence to legislate on Social Security matters is, and continues to be, the exclusive competence of the State. And, on the other hand, to verify how the last reforms in the field of pensions obey to national legislative initiatives, in no way imposed by European Law or international Law.

Keywords: European Union, social security, contributory old-age benefits.

Sumario: I. Introducción. II. La Seguridad Social en el Derecho originario de la Unión Europea. III. Competencias de la Unión Europea en materia de Seguridad Social. 1. Coordinación de sistemas de Seguridad Social. 2. Método abierto de coordinación. IV. Derecho de la Unión Europea y control del déficit público. 1. El Pacto de Estabilidad y Crecimiento. 2. Los Semestres Europeos. 3. Recomendaciones del Consejo de la Unión Europea a España en materia de Seguridad Social. A) Primera fase. B) Segunda fase. V. El control del gasto público y el Derecho Internacional. 1. El Tratado de "Estabilidad, Coordinación y Gobernanza" y el Tratado "Constitutivo del Mecanismo Europeo de Estabilidad". A) El principio de estabilidad presupuestaria y la reforma del artículo 135 de la Constitución Española. VI. Recapitulación: la legislación de Seguridad Social, competencia nacional. VII. Conclusiones.

\section{Introducción}

1. El presupuesto de la Seguridad Social, y el de las pensiones contributivas en particular, constituyen el capítulo más importante de los Presupuestos Generales del Estado ${ }^{1}$

2. La crisis económica y financiera internacional de 2008 afectó gravemente a las finanzas públicas españolas, con un espectacular incremento de la tasa de paro a lo que se unió el problema de la deuda soberana en la zona euro, que incrementó el diferencial de rendimiento de los títulos públicos. El déficit público en el año 2009 fue del 11,1\% del PIB.

3. Entre el amplio abanico de medidas adoptadas por España para reducir el gasto público interesa aquí destacar la reforma paramétrica de la legislación de pensiones de jubilación contributivas.

4. Existe una generalizada creencia -en no pocas ocasiones alentada por la clase política y expandida por los medios de comunicación- según la cual las reformas en materia de pensiones son consecuencia de imposiciones provenientes de las instituciones europeas, específicamente de las Recomendaciones anuales del Consejo de la Unión Europea a España. De esta manera, el ser un Estado miembro de la UE se utiliza a modo de "coartada" para legitimizar decisiones políticas que causarían de otro modo un rechazo social aun mayor, ya que los ciudadanos acaban por asumir lo que parece ser algo ineludible.

5. Paralelamente, y con la finalidad de corregir el déficit público excesivo que pone en riesgo la estabilidad de la moneda común (el euro), nuestro país ratificó en el último lustro dos importantes Tratados Internacionales de los que son parte los Estados de la UE: el Tratado de "Estabilidad, Coordinación y Gobernanza" y el Tratado del "Mecanismo Europeo de Estabilidad".

6. Para poder comprender la política de reformas restrictivas en el ámbito de las pensiones contributivas emprendida por España hay que tener presente que la misma es el resultado de la interacción de dos fuentes diferentes: el Derecho de la Unión Europea y el Derecho internacional. Ambas, sin embargo, confluyen en un objetivo común: dotar a la zona euro de estabilidad financiera.

7. En este trabajo se abordará desde una perspectiva jurídica la cuestión de si las reformas en materia de pensiones contributivas acometidas por España desde 2011 son fruto de la aplicación del

\footnotetext{
${ }^{1}$ En 2017 se destinaron al pago de pensiones contributivas 122.777 millones de euros; a pensiones no contributivas 2.320 millones de euros; 6.986 millones a prestaciones por Incapacidad Temporal y 2.450 millones a prestaciones por maternidad, paternidad, riesgos durante el embarazo y durante la lactancia natural y subsidio por cuidado de menores afectados por cáncer $\mathrm{u}$ otra enfermedad grave.
} 
Derecho de la Unión Europea en nuestro país, o impuestas por Tratados internacionales ratificados por España, o, por el contrario, han sido adoptadas por iniciativa del legislador nacional en el ejercicio de sus competencias soberanas.

8. En aras de una mayor claridad expositiva, este artículo se estructura en dos grandes bloques: por un lado el análisis del Derecho de la Unión Europea y las propuestas de reformas en materia de pensiones emanadas del Consejo de la Unión Europea. De otro lado, los compromisos internacionales asumidos por España para controlar el déficit público.

9. En ambos casos el fin es el mismo: ajustar el déficit público del Estado español a los límites impuestos por el Tratado de Funcionamiento de la Unión Europea y a los vigentes Tratados Internacionales, lo que exige una reducción del gasto público. Pero -y es lo que se pretende probar con este artículo- dejando plena libertad al Estado para lograr este fin, por lo que la reforma de la pensión contributiva de jubilación no tendría que haber sido, necesariamente, tan drástica y restrictiva como la resultante de la promulgación de la Ley 27/2011 sobre Actualización, Adecuación y Modernización del sistema de Seguridad Social; y de la Ley 23/2013 reguladora del Factor de Sostenibilidad y del Índice de Revalorización del Sistema de Pensiones de la Seguridad Social.

\section{La Seguridad Social en el Derecho originario de la Unión Europea}

10. En diversos preceptos del Tratado de Funcionamiento de la Unión Europea (TFUE) y en sus Protocolos hay referencias al término Seguridad Social, aunque en ninguno de ellos encontramos una definición legal de tal concepto².

11. Por su parte, el artículo 34 de la Carta de los Derechos Fundamentales de la Unión Europea ${ }^{3}$ lleva por rúbrica "Seguridad social y ayuda social". El citado precepto dispone que:

1. La Unión reconoce y respeta el derecho de acceso a las prestaciones de seguridad social y a los servicios sociales que garantizan una protección en casos como la maternidad, la enfermedad, los accidentes laborales, la dependencia o la vejez, así como en caso de pérdida de empleo, según las modalidades establecidas por el Derecho comunitario y las legislaciones y prácticas nacionales.

2. Toda persona que resida y se desplace legalmente dentro de la Unión tiene derecho a las prestaciones de seguridad social y a las ventajas sociales con arreglo al Derecho comunitario y a las legislaciones y prácticas nacionales.

3. Con el fin de combatir la exclusión social y la pobreza, la Unión reconoce y respeta el derecho a una ayuda social y a una ayuda de vivienda para garantizar una existencia digna a todos aquellos que no dispongan de recursos suficientes, según las modalidades establecidas por el Derecho comunitario y las legislaciones y prácticas nacionales".

12. Pero qué duda cabe que el término Seguridad Social a los efectos de la Carta resulta absolutamente confuso pues no permite diferencia entre Seguridad Social, "servicios sociales" y "ventajas sociales". Como tampoco resulta de utilidad para trazar la frontera entre prestaciones no contributivas de Seguridad Social y prestaciones no contributivas de Asistencia Social ${ }^{4}$, cuestión clave en España dada la distribución de competencias que la Constitución realizar en los artículos 147.1. 17 y 148.1.20, respectivamente.

\footnotetext{
${ }^{2}$ A título ilustrativo, cfr. artículos 21.3, 48, 153.1.c), 153.4 y 156 TFUE.

${ }^{3} \mathrm{Su}$ carácter de Derecho originario es indiscutible puesto que el artículo 6.1 de la versión consolidada del Tratado de la Unión Europea -DOUE 7.6.2016- afirma que "tendrá el mismo valor jurídico que los Tratados".

${ }^{4}$ STC 239/2002:"una interpretación del artículo 41 de la Constitución en el marco del bloque de constitucionalidad, permite inferir la existencia de una asistencia social "interna" al sistema de Seguridad Social y otra "externa" de competencia exclusiva de las Comunidades Autónomas". STC 76/1986: "en el momento actual -con independencia de que la evolución del sistema de Seguridad Social pueda ir en la misma dirección- es característica de la asistencia social su sostenimiento al margen de toda obligación contributiva o previa colaboración económica de los destinatarios o beneficiarios".
} 
13. En la práctica, el concepto de Seguridad Social ha devenido en un concepto de delimitación jurisprudencial por el máximo intérprete del Derecho de la UE: el Tribunal de Justicia con sede en Luxemburgo que, a su vez, le dota de distinto alcance según se trate de aplicar el Reglamento 888/2004 de coordinación de sistemas de Seguridad Social o la Directiva 2004/38.

\section{Competencias de la Unión Europea en materia de Seguridad Social}

14. Puesto que la UE no es un Estado ni tampoco una Organización Internacional al uso, su origen convencional "la somete al principio atributivo o de competencias de atribución. No hay ninguna facultad consustancial a su existencia, sino que toda su capacidad de actuar se deriva de lo recibido de sus Estados miembros por obra de los Tratados" $"$.

15. Es por ello necesario distinguir entre materias que son competencia exclusiva de los Estados miembros; materias atribuidas a la Unión Europea y un tercer grupo de competencias compartidas entre la UE y los Estados miembros.

\section{Coordinación de sistemas de Seguridad Social}

16. La legislación en materia de Seguridad Social no se encuentra entre las competencias nacionales atribuidas a la UE. Es por ello que, actualmente, coexisten en la UE tantos sistemas de Seguridad Social como Estados en los que el Derecho de la Unión Europea resulta aplicable. Cada sistema, fruto de un precipitado histórico, mantiene sus singularidades y diferencias respecto a las vías de financiación, ámbito de aplicación material y personal y procedimiento. Es decir, que no existe un sistema unificado y común de Seguridad Social en la UE.

17. Pero a fin de que esta multiplicidad de sistemas de Seguridad Social no constituyan un obstáculo insalvable para el ejercicio de unos de los pilares de la Unión Europea -la libre circulación de trabajadores- el TFUE regula en su artículo 48 la técnica de la coordinación entre sistemas de Seguridad Social (desarrollado por el vigente Reglamento 883/2004 y su Reglamento de Aplicación 987/2009) en los siguientes términos:

18. El Parlamento Europeo y el Consejo, con arreglo al procedimiento legislativo ordinario, adoptarán, en materia de seguridad social, las medidas necesarias para el establecimiento de la libre circulación de los trabajadores, creando, en especial, un sistema que permita garantizar a los trabajadores migrantes por cuenta ajena y por cuenta propia, así como a sus derechohabientes:

a) la acumulación de todos los periodos tomados en consideración por las distintas legislaciones nacionales para adquirir y conservar el derecho a las prestaciones sociales, así como para el cálculo de éstas;

b) el pago de las prestaciones a las personas que residan en los territorios de los Estados miembros.

19. La coordinación se limita a establecer los criterios de conexión entre las diferentes legislaciones nacionales así como de distribución de las cargas entre los distintos regímenes nacionales, sin que los derechos en materia de Seguridad Social de los migrantes se vean afectados por su lugar de empleo o residencia.

\footnotetext{
5 J. M. Miranda Boto, Las Competencias de la Comunidad Europea en Materia Social, Thomson-Reuter, Pamplona, 2009, p.39.
} 
20. No fue concebida como un objetivo en si mismo, sino que como afirma MIRANDA BOTO "nació como un simple complemento de la libre circulación" por lo que tiene un carácter meramente instrumental.

21. La coordinación se articula en torno a cinco pilares regulados en el Reglamento 883/2004: igualdad de trato (artículo 4); totalización de períodos (artículo 6); asimilación de prestaciones, ingresos, hechos o acontecimientos (artículo 5); supresión de cláusulas de residencia (artículo 7); y unicidad de la legislación aplicable (artículo 11).

22. No existe, sin embargo, una definición del término "coordinación" ni en el Derecho originario de la Unión Europea ni en el Derecho derivado, de ahí que resulte obligada la remisión a la jurisprudencia del Tribunal de Justicia de la Unión Europea de la que se extraen las siguientes conclusiones:

- La coordinación no restringe la competencia de los Estados miembros para regular sus sistemas de Seguridad Social, que siguen subsistiendo con todas sus peculiaridades y divergencias de procedimiento.

- La coordinación no altera las diferencias de los derechos de las personas que trabajan en dichos Estados.

- La coordinación no supone suprimir las diferencias de fondo entre los regímenes de Seguridad Social de los Estados miembros.

- La coordinación no implica la uniformización de los sistemas, ni mucho menos la unificación.

\section{Método abierto de coordinación}

23. Una de las novedades introducidas por el Tratado de Lisboa fue la instauración del Método Abierto de Coordinación, que no debe ser confundido con la técnica de coordinación de sistemas de Seguridad Social descrita en el epígrafe precedente.

24. El Método Abierto de Coordinación tiene por objetivo "establecer directrices para la Unión combinadas con calendarios específicos para lograr los objetivos que se fijen a corto, medio y largo plazo; establecer, cuando proceda, indicadores y puntos de referencia cuantitativos y cualitativos cotejados con los mejores que existan y adaptados a las necesidades de los distintos Estados miembros y sectores como método de comparación de las prácticas idóneas; plasmar estas directrices europeas en medidas de política nacional; y organizar periódicamente controles y evaluaciones entre homólogos como procesos de aprendizaje mutuo"7.

25. El Método Abierto de Coordinación pretende "intercambiar experiencias y buenas prácticas, coordinar políticas sociales y desarrollar una cooperación interestatal más intensa. Las materias que abarca son pensiones, la asistencia sanitaria y la atención de larga duración, además de la inclusión social. El órgano de debate, el Comité de Protección Social. Los instrumentos, informes de estrategia nacionales"8.

26. El artículo 160 del TFUE regula el Comité de Protección Social. Es un órgano de carácter consultivo que tiene por finalidad fomentar la cooperación en materia de protección social entre los Estados miembros y con la Comisión. El Comité tendrá por misión: supervisar la situación social y la evolución de las políticas de protección social de los Estados miembros y de la Unión; facilitar el inter-

\footnotetext{
${ }^{6}$ J.M. MiRanda Boto, Las Competencias de la Comunidad Europea en Materia Social, Op. Cit., p. 264.

${ }^{7}$ V. Pérez Menayo, "El Método Abierto de Coordinación en la Unión Europea: su Aplicación a las Pensiones", Noticias de la Unión Europea, $\mathrm{n}^{\mathrm{0}} 222,2003$, p.52.

${ }^{8}$ C. García de Cortázar Nebrada, "Panorama de la Seguridad Social en las Comunidades Europeas (1997-2002)", Noticias de la Unión Europea, $\mathrm{n}^{\mathrm{o}} 222,2003, \mathrm{p} .15$
} 
cambio de información, experiencias y buenas prácticas entre los Estados miembros y con la Comisión, y elaborar informes, emitir dictámenes o emprender otras actividades en los ámbitos que sean de su competencia, ya sea a petición del Consejo o de la Comisión, ya por propia iniciativa.

27. A través del Método Abierto de Coordinación la UE respalda las reformas de los Estados miembros "mediante la definición de objetivos comunes y un proceso de aprendizaje mutuo. Concretamente, ofrece a los a los Estados miembros: objetivos comunes; indicadores comunes para medir los progresos: un marco de presentación de informes; e indicadores de referencia para comparar los resultados e identificar las mejores prácticas"

28. En el ámbito concreto de las pensiones, el Método Abierto de Coordinación se limita a "fijar un marco común para que los Estados miembros compartan ideas, enfoques, conocimientos y experiencias en materia de pensiones centrados en los siguientes objetivos comunes: ingresos de jubilación adecuados para todos, teniendo en cuenta la solidaridad y la equidad entre generaciones; sostenibilidad financiera de los sistemas de pensiones públicos y privados, en particular, fomentando la prolongación de la vida laboral y el envejecimiento activo; y sistemas de pensiones transparentes y debidamente adaptados a las necesidades individuales y sociales"10.

29. Pero, al objeto de este estudio, resulta absolutamente necesario destacar que este Método Abierto de Coordinación no afecta en absoluto a la competencia exclusiva de los Estados miembros en materia de legislación nacional de Seguridad Social.

30. Son los Estados, y solo los Estados, "los principales responsables del diseño de sus sistemas de pensiones de acuerdo con sus realidades y contextos nacionales" $"$.

\section{Derecho de la Unión Europea y control de déficit público}

31. A pesar de lo expuesto en las páginas precedentes, tras la puesta en marcha de los Semestres Europeos en 2011 hay que replantearse si la legislación en materia de Seguridad Social, y de las pensiones en particular, sigue siendo competencia exclusiva de los Estados miembros.

32. Y es que desde 2011, a través de las Recomendaciones del Consejo de la Unión Europea, a cada país se le dirigen recomendaciones específicas que "no sólo reflejan el programa económico y social de la Comisión Europea, sino que incorporan reformas en materia de pensiones"12.

33. El origen de esta situación radica en que conforme al TFUE es preciso distinguir entre la política monetaria (competencia de la EU de conformidad con su artículo $3.4^{13}$ ) y la política económica ${ }^{14}$ (basada en la coordinación de las políticas económicas de los Estados miembros ex artículo $119^{15}$ ).

\footnotetext{
${ }^{9}$ Comisión Europea, La Aportación de la Unión Europea al Envejecimiento Activo y la Solidaridad entre las Generaciones, Bruselas, 2012, p. 14.

${ }^{10}$ Comisión Europea, La Aportación de la Unión Europea al Envejecimiento Activo y la Solidaridad entre las Generaciones, Op. Cit., p. 14.

${ }^{11}$ I. Camós Victoria, C. García de Cortázar, y B. Suárez Corujo, La Reforma de los Sistemas de Pensiones en Europa, Laborum. 2017, p. 26.

${ }^{12}$ I. Camós Victoria, C. García de Cortázar, y B. Suárez Corujo, La Reforma de los Sistemas de Pensiones en Europa, Op. Cit., p. 26.

13 "La Unión establecerá una unión económica y monetaria cuya moneda es el euro".

${ }^{14}$ A. Aguilar Calahorro, "La Decisión Pringle en el Proceso de Constitucionalización de la Unión Europea", Revista Española de Derecho Constitucional, $n^{\circ} 101,2014$, p.350.

15 "Para alcanzar los fines enunciados en el artículo 3 del Tratado de la Unión Europea, la acción de los Estados miembros y de la Unión incluirá, en las condiciones previstas en los Tratados, la adopción de una política económica que se basará en la estrecha coordinación de las políticas económicas de los Estados miembros, en el mercado interior y en la definición de objetivos comunes, y que se llevará a cabo de conformidad con el respeto al principio de una economía de mercado abierta y de libre competencia”.
} 
34. Con el Tratado de Maastricht se sentaron las bases para una Unión Monetaria que se materializó con la instauración de una moneda común (el euro), cuya estabilidad requiere un férreo control por parte de los Estados de la eurozona del déficit público.

35. El TFUE -artículo 104 del TCE, actual 126 del TFUE- impone a los Estados evitar déficit públicos excesivos. En concreto es su Protocolo n ${ }^{\circ}$ 12, "Sobre el Procedimiento Aplicable en Caso de Déficit Excesivo"16, el que especifica los valores que se mencionan en el artículo 126.2 TFUE"

- 3\% en lo referente a la proporción entre el déficit público o real y el PIB a precios de mercado

- $60 \%$ en lo referente a la proporción entre la deuda pública y el PIB a precios de mercado.

36. Como ha reconocido la propia Comisión Europea en su Libro Blanco (2012), las reformas de jubilación en los Estados miembros se consideran un factor determinante para el buen funcionamiento de la Unión Económica y Monetaria ${ }^{18}$.

37. Para comprender el cómo y por qué la adopción de la moneda única ha influenciado en las reformas paramétricas de las pensiones contributivas promulgadas en España tras la última crisis económica y financiera mundial es preciso hacer referencia -aunque sea esquemáticamente- a conceptos e instrumentos jurídicos ajenos al Derecho de la Seguridad Social.

38. Pero sin estas nociones previas no es posible afrontar la cuestión de si el Consejo de la Unión Europea se ha extralimitado al hacer recomendaciones específicas a España entre los años 2011-2013 en materia de reforma de pensiones que han sido acatadas por España como de obligado cumplimiento.

\section{El Pacto de Estabilidad y Crecimiento}

39. A fin de hacer respetar las obligaciones asumidas por los Estados miembros en materia de déficit se aprobó en 1997 el Pacto de Estabilidad y Crecimiento (PEC) -que ha sido a su vez objeto de diversas reformas ${ }^{19}-$.

40. El PEC ha sido definido como "un conjunto de normas para garantizar que los países de la Unión Europea mantengan unas finanzas públicas saneadas y coordinar sus políticas presupuestarias".

\footnotetext{
${ }^{16}$ DOUE C 306/1, de 17.12.2007.

17 "La Comisión supervisará la evolución de la situación presupuestaria y del nivel de endeudamiento público de los Estados miembros con el fin de detectar errores manifiestos. En particular, examinará la observancia de la disciplina presupuestaria atendiendo a los dos criterios siguientes:

a) si la proporción entre el déficit público previsto o real y el producto interior bruto sobrepasa un valor de referencia, a menos:

— que la proporción haya descendido sustancial y continuadamente y llegado a un nivel que se aproxime al valor de referencia,

— que el valor de referencia se sobrepase sólo excepcional y temporalmente, y la proporción se mantenga cercana al valor de referencia;

b) si la proporción entre la deuda pública y el producto interior bruto rebasa un valor de referencia, a menos que la proporción disminuya suficientemente y se aproxime a un ritmo satisfactorio al valor de referencia.

Los valores de referencia se especifican en el Protocolo sobre el procedimiento aplicable en caso de déficit excesivo, anejo a los Tratados"

${ }^{18}$ Comisión Europea, Libro Blanco “Agendas para unas Pensiones Adecuadas, Seguras y Sostenibles”, Bruselas, 2012, p.3.

${ }^{19}$ El 13 de diciembre de 2011 entró en vigor el primer conjunto de medidas de gobernanza económica (six-pack), que reforma y modifica las normas del Pacto de Estabilidad y Crecimiento. El PEC modificado establece los principales instrumentos para la supervisión de las políticas presupuestarias de los Estados miembros (vertiente preventiva) y para la corrección del déficit excesivo (vertiente correctora).
} 
41. Sobre el PEC en el "Libro Verde" 20 se afirma que "ofrece el marco para hacer un seguimiento de la sostenibilidad de las finanzas públicas, incluidos los sistemas de pensiones".

42. El PEC prevé la posibilidad de imponer sanciones pecuniarias a los Estados cuando los mismos no adopten las medidas de ajuste pertinentes, o cuando los Estados manipulen datos relativos al déficit o a la deuda. Por lo que respecta a España se le ha llegado a abrir expediente sancionador por déficit excesivo.

43. El PEC tiene una vertiente preventiva con fundamento en el artículo 121 TFUE (supervisión de las políticas presupuestarias de los Estados para garantizar la solidez de las finanzas públicas); y una vertiente correctora basada en el artículo 126 TFUE (corrección del déficit excesivo).

\section{Los Semestres Europeos}

44. El Semestre Europeo se creó en 2010 por el Consejo Europeo. El primero tuvo lugar en 2011.

45. El Semestre Europeo combina "la coordinación de la política presupuestaria, regida por el Pacto de Estabilidad y Crecimiento, con la coordinación de las políticas económicas, estructurales y de empleo, regidas por la estrategia de crecimiento Europa 2020"21.

46. El Semestre Europeo incluye tres áreas de coordinación de políticas económicas: "reformas estructurales, dedicadas a promover el crecimiento y el empleo de conformidad con la Estrategia Europa 2020; políticas presupuestarias, para garantizar la sostenibilidad de la hacienda pública de conformidad con el Pacto de Estabilidad y Crecimiento; y prevención de desequilibrios macroeconómicos excesivos".

47. Se pretende, en definitiva, llevar a cabo un seguimiento y análisis de los desequilibrios macroeconómicos, emitiéndose recomendaciones a los Estados en caso necesario para que los corrijan.

48. En el marco de los Semestres Europeos se evalúan los Programas Nacionales de Reformas que presentan los Estados miembros y que tienen por objetivo definir su plan de reformas estructurales para promover el crecimiento y el empleo en aras de la consecución de la Estrategia Europa 2020.

49. Igualmente, son objeto de evaluación en los Semestres Europeos los Programas Nacionales de Estabilidad, que se enmarcan en la vertiente preventiva del Pacto de Estabilidad y Crecimiento. En ellos se plasman las estrategias presupuestarias a medio plazo nacionales ${ }^{22}$.

50. Los Programas de Estabilidad tienen la consideración de plan fiscal nacional a medio plazo al que hace referencia el artículo 4 del Reglamento (UE) 473/2013. Han de ser presentados anualmente por los Estados de la zona euro. Su objetivo el garantizar la estabilidad presupuestaria dentro de la zona de la moneda única.

\footnotetext{
${ }^{20}$ Comisión Europea, Libro Verde "En pos de unos sistemas de pensiones europeos adecuados, sostenibles y seguros", Bruselas, 2010, p.10.

"Los Libros Verdes son documentos publicados por la Comisión Europea cuyo objetivo es estimular una reflexión a nivel europeo sobre un tema concreto. Los Libros Verdes invitan a las partes interesadas (organism2os y particulares) a participar en un proceso de consulta y debate sobre las propuestas que presentan, y pueden dar origen a desarrollos legislativos que se reflejan en Libros Blancos".

${ }^{21}$ A. BAR Cendrón, "La Reforma Constitucional y la Gobernanza Económica de la Unión Europea", Teoría y Realidad Constitucional, $\mathrm{n}^{\mathrm{o}} 30,2012$, p.73.

22 "Los programas de estabilidad han de incluir, entre otros aspectos, el objetivo presupuestario a medio plazo, la trayectoria de ajuste hacia dicho objetivo y un análisis de sucesos en el que se examinen los efectos de los cambios en los principales supuestos económicos subyacentes sobre la situación fiscal. Los cálculos deben basarse en los sucesos macrofiscales más probables (o más prudentes)".
} 
51. A fin de una mayor efectividad y coordinación, el Programa de Estabilidad se presenta coetáneamente con el Programa Nacional de Reformas.

52. Una vez evaluados los Programas Nacionales de Reformas y los Programas de Estabilidad por la Comisión Europea, ésta última elabora recomendaciones individualizadas para cada Estado miembro que, tras la oportuna tramitación, habrán de ser finalmente adoptadas por el Consejo de la Unión Europea en el mes de julio.

53. Las Recomendaciones del Consejo de la Unión Europea son documentos en los que se analiza la situación económica de cada Estado miembro y se recomiendan medidas que cada Estado debe tomar en los doce meses siguientes. Su ámbito puede ser muy heterogéneo, pero interesa destacar que las Recomendaciones específicas han incluido acciones correctoras también en el ámbito de la Seguridad Social.

\section{Recomendaciones del Consejo de la Unión Europea en materia de Seguridad Social}

54. Las Recomendaciones específicas -enmarcadas en el Semestre Europeo- "al incorporar reformas en materia de pensiones incluyen acciones, medidas a desarrollar directamente por los Estados miembros" 23 .

55. Es por ello que debemos preguntarnos si, a través de las Recomendaciones específicas que desde 2011 anualmente el Consejo de la Unión Europea hace a los Estados miembros podría estar invadiendo competencias nacionales en materia de legislación de Seguridad Social, al menos por lo que a España se refiere.

56. Para poder responder a esta pregunta es preciso realizar en primer lugar un análisis de los Planes de Estabilidad y Planes de Reforma presentados por España desde 2011. Y confrontarlos con las correspondientes Recomendaciones específicas del Consejo de la Unión Europea para nuestro país.

57. Se trata, en síntesis, de comprobar si existe una correlación entre las primeras y la segunda, de manera que el Consejo de la Unión Europea se limita, o no, a respaldar las propuestas de reformas que el propio Estado le presenta. O si por el contrario el Consejo de la Unión Europea por propia iniciativa marca una "hoja de ruta" en cuanto a las reformas concretas que el Estado ha de acometer.

58. Tras un análisis pormenorizado de las disposiciones mencionadas lo primeo que se constata es que se pueden distinguir dos fases bien diferenciadas:

\section{A) Primera fase}

59. La primera fase comprende los años 2011, 2012 y 2013. Este trienio tiene como elemento en común que en todos estos años las Recomendaciones específicas a España contienen expresas referencias a la Seguridad Social y a la reforma de las pensiones en particular.

\section{Año 2011}

60. El 29 de abril España presentó la actualización de su Programa de Estabilidad, que parte de la premisa de que "la situación actual del Sistema de la Seguridad Social es sólida, presentando un superávit en 2010 (excluido el Servicio Público de Empleo) y contando con un fondo de reserva que supone el $6,1 \%$ del PIB".

${ }^{23}$ I. Camós Victoria, C. García de Cortázar y B. Suárez Corujo, La Reforma de los Sistemas de Pensiones en Europa, Op. cit.; p.26. 
61. Pese a tan optimista afirmación, en el capítulo 8 del Plan se detallan las reformas del sistema público de pensiones que el Gobierno ha impulsado, entre las que destacan las siguientes:

- Aumento gradual de la edad legal de jubilación hasta alcanzar los 67 años, excepto para las carreras largas.

- Endurecimiento de las condiciones para el acceso a la jubilación anticipada y parcial.

- Ampliación del periodo de cómputo de la pensión de los últimos 15 años a los últimos 25.

- El periodo cotizado para tener derecho al 100\% de la pensión pasa de 35 a 37 años.

- Incrementar los incentivos para la prolongación voluntaria de la vida laboral más allá de la edad legal de jubilación.

— Introducción de un factor de sostenibilidad.

62. Reformas que se recogen en la Ley 27/2011, sobre Actualización, Adecuación y Modernización del Sistema de Seguridad Social. Y que como en su Preámbulo se destaca, fue fruto del consenso social: incorpora los compromisos recogidos en el Acuerdo Social y Económico para el crecimiento, el empleo y la garantía de las pensiones, de 2 de febrero de 2011. La Ley 27/2011 entró en vigor el 1 de enero de 2013.

63. Por su parte, el Plan de Reforma del año 2011, presentado el 29 de abril, se remite al Plan de Estabilidad por lo que a la reforma del sistema de pensiones se refiere.

64. En la segunda de las siete admoniciones que contiene la Recomendación del Consejo de la Unión Europea de 12.7.2011 a España ${ }^{24}$ se recomienda "adoptar la reforma del sistema de pensiones propuesta con el fin de retrasar la edad de jubilación legal y aumentar el número de años de trabajo para el cálculo de las pensiones, según lo planeado; revisar periódicamente los parámetros de las pensiones a la luz de la evolución de la esperanza de vida, según lo previsto, y adoptar nuevas medidas encaminadas a mejorar el aprendizaje permanente para los trabajadores de más edad".

65. Esta última propuesta, como GUTIÉRREZ BENGOECHEA pone de manifiesto ${ }^{25}$, se materializaría con la aprobación del Real Decreto Ley 5/2013, de Medidas para Favorecer la Continuidad de la Vida Laboral de los Trabajadores de Mayor Edad y Promover el Envejecimiento Activo.

66. La conclusión que se alcanza es que la iniciativa, contenido y alcance de la reforma del sistema de pensiones es imputable al Gobierno, respaldado por lo que se refiere a la Ley 27/2011 por el consenso social. En ningún caso ordenada o impuesta por el Consejo de la UE que se limita a refrendar la propuesta que el propio Estado presenta dentro de su política de contención del gasto público.

67. Las recomendaciones específicas de 2011 del Consejo de la Unión Europea a España (y al resto de los Estados miembros) aparecen transcritas en el Anexo III del Libro Blanco "Agenda para unas Pensiones Adecuadas, Seguras y Sostenibles".

68. Hay que descartar la naturaleza de fuente del Derecho en materia de reforma de pensiones del citado Libro Blanco. Y precisamente porque las Recomendaciones específicas a cada Estado por parte del Consejo de la Unión Europea difieren notablemente en cuanto a contenido dependiendo del país, es por lo que siendo aparentemente similares los problemas que los sistemas públicos de pensiones europeos han de afrontar, este Anexo III del Libro Blanco tiene un contenido tan dispar.

\footnotetext{
${ }^{24}$ DOUE de 19 de julio de 2011, C 212/01.

${ }^{25}$ M. Gutiérrez Bengoechea, "La Sostenibilidad de las Pensiones Públicas en un Estado de Bienestar Social”, E-Revista Internacional de la Protección Social, vol.2 n², 2017, p.102.
} 


\section{Año 2012}

69. España presentó el 30 de abril su Programa de Estabilidad, que prevé que el gasto en pensiones entre 2010 y 2060 aumente " 3,6 puntos de PIB, frente a los 6,6 puntos en un escenario sin reforma, por lo que el ahorro estimado de la reforma, excluido el factor de sostenibilidad, asciende a 3 puntos de PIB. Este ahorro se explica fundamentalmente por el alargamiento de la vida laboral. La ampliación de la edad de jubilación tiene un doble efecto positivo. Reduce la ratio de pensionistas sobre la población de más de 65 años, y aumenta la tasa de empleo de los trabajadores de mayor edad".

70. Entre los hitos destacados por el Plan de Reforma, de 30 de abril, se encuentra el dato de la promulgación de la Ley 27/2011, de 1 de agosto, de conformidad con la segunda recomendación del Consejo de la Unión Europea de 12.7.2011. Esta reforma trajo consigo, entre otros efectos, el incremento de la edad de jubilación, afectó al cálculo de la base reguladora y a la escala para obtener el cien por cien de la base reguladora e, igualmente, modifico el acceso a la jubilación parcial y anticipada ${ }^{26}$.

71. En el undécimo Considerando de las Recomendaciones a España del Consejo de la Unión Europea de 10.7.2012 ${ }^{27}$ se hace constar que "en 2011, España adoptó una reforma de las pensiones que representa un paso significativo en favor de la sostenibilidad a largo plazo de la hacienda pública. Sin embargo, el deterioro de las perspectivas de la economía española está limitando los efectos de la reforma sobre la previsión de gastos públicos que se derivarían del envejecimiento de la población. Por otra parte, la reforma aún necesita complementarse con medidas concretas encaminadas a sostener la Estrategia global para el empleo de los trabajadores y las trabajadoras de más edad 2012- 2014".

72. Y, específicamente en materia de pensiones, la segunda recomendación del Consejo de la Unión Europea es: "asegurar que la edad de jubilación vaya aumentando en función de la esperanza de vida a la hora de regular el factor de sostenibilidad previsto en la reciente reforma del sistema de pensiones y respaldar la Estrategia global para el empleo de los trabajadores y las trabajadoras de más edad con medidas concretas encaminadas a desarrollar más el aprendizaje permanente, mejorar las condiciones laborales y fomentar la reincorporación de este grupo de trabajadores al mercado de trabajo".

\section{Año 2013}

73. En la Actualización del Programa de Estabilidad presentado por España 30 de abril destaca la afirmación de que "la relevancia del factor de sostenibilidad ha quedado reforzada en la Ley Orgánica 2/2012, de 27 de abril, de Estabilidad Presupuestaria y Sostenibilidad Financiera, cuyo artículo 18.3 prevé que el Gobierno, en caso de proyectar un déficit de largo plazo del sistema de pensiones, revisará el sistema aplicando de forma automática el factor de sostenibilidad en los términos y condiciones previstos en la Ley 27/2011, de 1 de agosto, sobre actualización, adecuación y modernización del sistema de Seguridad Social". La reforma del 2011 "se complementa con una segunda etapa de reformas que incluyen las medidas en materia de jubilación anticipada y parcial y de fomento de la compatibilización entre trabajo y pensión contenidas en el Real Decreto-ley $5 / 2013^{28}$, de 15 de marzo, de medidas para favorecer la continuidad de la vida laboral de los trabajadores de mayor edad y promover el envejecimiento activo".

74. En el Programa Nacional de Reformas de 2013, presentado el 30 de abril, se cuantifica el ahorro esperado en el PIB tras la adopción de las reformas contenidas en el Real Decreto Ley 5/2013 y de los resultados del grupo de trabajo creado para diseñar el factor de sostenibilidad.

\footnotetext{
${ }^{26}$ Cfr. C. Gala Durán, "La reforma de la pensión de jubilación en las leyes 27/2011 y 3/2012: avances hacia un nuevo modelo", Revista Internacional de Organizaciones, n 8, 2012, p. 90.

${ }^{27}$ DOUE de 24 de julio de 2012, C 219/81.

${ }^{28}$ Esta norma introdujo reformas que afectaron a la jubilación anticipada y a la jubilación parcial. Penaliza a las empresas que despiden a los trabajadores de más edad. Y amplió la posibilidad de compatibilizar trabajo y pensión de jubilación, para incentivar la permanencia en el mercado de los trabajadores que hayan alcanzado la edad ordinaria de jubilación.
} 
75. La Recomendación del Consejo de la Unión Europea de 9.7.2013 $3^{29}$ contiene nueve admoniciones. En la primera de ellas in fine se lee: "culminar al final de 2013 a más tardar la regulación del factor de sostenibilidad a fin de garantizar la estabilidad financiera a largo plazo del sistema de pensiones incluso aumentando la edad efectiva de jubilación mediante el ajuste de la edad de jubilación o las pensiones de jubilación a los cambios en la esperanza de vida".

\section{B) Segunda fase}

76. Las Recomendaciones específicas correspondientes a los años 2014 a 2017 se caracterizan por la ausencia de previsiones específicas en materia de reformas de pensiones.

\section{Año 2014}

77. En la Actualización del Programa de Estabilidad presentada el 30 de abril se destaca cómo en 2013 se adoptaron "dos importantes reformas: por un lado, el Real Decreto Ley 5/2013, de medidas para favorecer la continuidad de la vida laboral de los trabajadores de mayor edad y promover el envejecimiento activo; y por otro, la Ley 23/2013, de 23 de diciembre, reguladora del factor de sostenibilidad y del índice de revalorización del sistema de pensiones". Y "como consecuencia de las reformas de pensiones adoptadas por España en 2013, el indicador de sostenibilidad S2 mejorará sensiblemente".

78. Respecto al Fondo de Reserva de la Seguridad Social el citado documento constata que "en diciembre de 2013 cuenta con 53.744 millones de euros (5,25\% del PIB)".

79. Pero, pesar de las reformas en materia de pensiones acometidas por el Gobierno hasta la fecha, "las pensiones contributivas tienen consignado un crédito para 2014 de 112.103 millones de euros, un $5,4 \%$ superior al del pasado año".

80. En el Anexo I del Plan de Reforma presentado por España el 30 de abril se contiene un cuadro explicativo de la aplicación de las Recomendaciones específicas recibidas del Consejo de la Unión Europea y las medidas adoptadas.

81. De las ocho admoniciones que contiene la Recomendación del Consejo de la Unión Europea de 8.7.2014 ${ }^{30}$ ninguna de ellas está directamente relacionada con el tema de las pensiones.

\section{Año 2015}

82. El 30 de abril de 2015 España presentó su Programa Nacional de Reformas y su Programa de Estabilidad.

83. En el primero de ellos se constata que "las pensiones contributivas tienen consignado un crédito para 2015 de 115.669 millones de euros, un 3,2\% superior al del pasado año, estimado en función del incremento del colectivo de pensionistas y de la variación de la pensión media y de una revalorización de un $0,25 \%$.". Por su parte, "las pensiones no contributivas de la Seguridad Social aumentan un 3,5\%".

84. El Programa Nacional de Reformas recoge en su Anexo I un cuadro con la aplicación de las recomendaciones específicas.

85. El Considerando $n^{\circ} 13$ de la Recomendación del Consejo de la Unión Europea de 14.7.2015 señala que:"España ha realizado avances limitados en la mejora de la eficacia de su sistema de protec-

\footnotetext{
${ }^{29}$ DOUE de 30 de julio de 2013, C 217/81.

${ }^{30}$ DOUE de 29 de julio de 2014, C 247/35.

${ }^{31}$ DOUE de 18 de agosto de 2015, C 272/46.
} 
ción social. Ha establecido un nuevo programa de activación para los desempleados de larga duración, que combina el apoyo a la renta con asistencia en la búsqueda de empleo. No obstante, la coordinación limitada entre los servicios de empleo y los servicios sociales y la incompatibilidad de los distintos regímenes de renta mínima han reducido la eficacia de los programas de ayuda social".

86. De las cuatro recomendaciones que contiene, ninguna específicamente afecta al tema de las pensiones o su reforma.

\section{Año 2016}

87. El 29 de abril de 2016, España presentó su Programa Nacional de Reformas y el 30 de abril de 2016, su Programa de Estabilidad.

88. En el Programa de Estabilidad se constata que "las pensiones contributivas tienen consignado un crédito para 2016 de 118.942 millones de euros, un 2,8\% superior al del pasado año y las pensiones no contributivas de la Seguridad Social aumentan un 2,2\%". Dado "la senda ascendente que sigue el gasto en pensiones, la Ley de Presupuestos Generales del Estado para 2016 incluyó una disposición para realizar, en el marco del Pacto de Toledo, un análisis que fortalezca las fuentes de financiación de la Seguridad Social y que profundice en el distinto carácter de las prestaciones contributivas y no contributivas".

89. En el Considerando $n^{0} 10$ de la Recomendación del Consejo de la Unión Europea de 12 de julio $^{32}$ se constata que "aunque el desempleo está disminuyendo rápidamente, sigue siendo muy elevado, en particular entre los jóvenes, y su duración media es también muy elevada. La persistencia de índices elevados de desempleo de larga duración implica que la falta de empleo puede hacerse crónica, en detrimento de las condiciones laborales y sociales. Casi el $60 \%$ de los parados de larga duración están poco cualificados,".

90. De las cuatro recomendaciones específicas que contiene este documento, ninguna afecta al tema de las pensiones o su reforma. En 2016 el gasto en pensiones ascendió a 134.820 millones de euros, lo que representa el 12,1 del PIB. Correlativamente el sistema de Seguridad Social tuvo un déficit de 18.096 millones de euros, equivalente al 1,6 PIB.

\section{Año 2017}

91. El 28 de abril de 2017, España presentó su Programa Nacional de Reformas de 2017 y el 29 de abril de 2017 su Programa de Estabilidad de 2017.

92. En el Anexo I del Programa de Reforma se recoge la aplicación de las recomendaciones específicas.

93. Ninguna de las tres recomendaciones específicas que contiene la Recomendación del Consejo de la Unión Europea a nuestro país de11.7.201733 afecta a las pensiones o su reforma. Y todo ello a pesar de que el gasto en pensiones ascendió a 134.820 millones de euros, lo que representa el 12,1 del PIB. Correlativamente el sistema de Seguridad Social tuvo un déficit de 18.096 millones de euros, equivalente al 1,6 PIB.

\footnotetext{
${ }^{32}$ DOUE de 18 de agosto de 2016, C 299/07.

${ }^{33}$ DOUE de 9 de agosto de 2017, C-261.
} 


\section{El control del gasto público y el Derecho Internacional}

\section{EI Tratado de "Estabilidad, Coordinación y Gobernanza" y el Tratado "Constitutivo del Meca- nismo Europeo de Estabilidad"}

94. La carencia por parte de la UE de "amplias competencias fiscales, de una política presupuestaria común y de una coordinación de las políticas económicas", unido "a una situación de crisis asimétricas e intereses (económicos y monetarios) contrapuestos entre los diferentes Estados que conforman esa Unión Económica Monetaria" 34 abocaron a la firma del Tratado Intergubernamental de "Estabilidad, Coordinación y Gobernanza en la Unión Económica y Monetaria" el 2 de marzo de 2012 y que entró en vigor 1 de enero de 2013 (también conocido como Pacto Presupuestario). Sólo tres Estados no lo han firmado: Reino Unido, República Checa y Croacia.

95. Su artículo 2.1 dispone que su aplicación no afectará a las competencias de la UE para actuar en el ámbito de la unión económica.

96. Para garantizar la efectividad de las reglas contenidas en el citado Tratado su artículo 3.2 exige su transposición al "Derecho nacional mediante disposiciones que tengan fuerza vinculante y sean de carácter permanente, preferentemente de rango constitucional, o cuyo respeto y cumplimiento estén de otro modo plenamente garantizados a lo largo de los procedimientos presupuestarios nacionales".

97. Aunque el Tratado de "Estabilidad, Coordinación y Gobernanza" regula materias que son competencia de la Unión Europea, desde el punto de vista de las fuentes del Derecho es digno de ser resaltado el hecho de que no estemos ante una norma de Derecho de la Unión Europea sino ante un Tratado Internacional. Ello es debido a que fue negociado al margen del procedimiento instaurado por el Derecho de la UE.

98. En España, y en aplicación del procedimiento establecido en el artículo 93 de la Constitución, fue la Ley Orgánica 3/2012 -BOE de 26.7.2012- la que autorizó la ratificación del Tratado de "Estabilidad, Coordinación y Gobernanza". En virtud del mismo, los Estados miembros de la EU firmantes acuerdan "reforzar el pilar económico de la unión económica y monetaria mediante la adopción de un conjunto de normas destinadas a promover la disciplina presupuestaria a través de un pacto presupuestario, a reforzar la coordinación de sus políticas económicas y a mejorar la gobernanza de la zona del euro".

99. Complementario al Tratado de "Estabilidad, Coordinación y Gobernanza" es el Tratado "Constitutivo del Mecanismo Europeo de Estabilidad" (MEDE), -hecho en Bruselas el 2.2.2012- del que son partes los Estados de la eurozona. Fue ratificado por España-BOE de 4.10.2012-. Puede ser descrito como "un sistema de concesión de ayudas financieras a Estados en situaciones de grave riesgo" 35 .

100. El MEDE fue incorporado al TFUE por medio de la Decisión 2011/199, que añadió al artículo 136 un nuevo apartado 3 que dispone que: "los Estados miembros cuya moneda es el euro podrán establecer un mecanismo de estabilidad que se activará cuando sea indispensable para salvaguardar la estabilidad de la zona del euro en su conjunto. La concesión de toda ayuda financiera necesaria con arreglo al mecanismo se supeditara a condiciones estrictas".

101. Sobre la competencia de los Estados miembros cuya moneda es el euro para establecer entre ellos un mecanismo de estabilidad a través de un Tratado internacional (MEDE) y plasmarlo en una

\footnotetext{
${ }^{34}$ J. Martín y Pérez de Nanclares, "El Nuevo Tratado de Estabilidad, Coordinación y Gobernanza en la Unión Económica y Monetaria: Reflexiones a propósito de una peculiar reforma realizada fuera de los Tratados Constitutivos", Revista de Derecho Comunitario Europeo, no 42,2012 , p. 402.

${ }^{35}$ A. Aguilar Calahorro, "La Decisión Pringle en el Proceso de Constitucionalización de la Unión Europea”. Op. cit.; p. 343.
} 
norma de Derecho comunitario se pronunció favorablemente el TJUE en su sentencia de 27.11.2012, $-370 / 12$, (Pringle) ${ }^{36}$.

102. La concesión de asistencia financiera a través del nuevo Tratado MEDE, estará condicionado a partir de marzo 2013 a la ratificación del Tratado de "Estabilidad, Coordinación y Gobernanza" por el Estado miembro del MEDE de que se trate.

103. Como conclusión provisional, y siguiendo a SERRANO PÉREZ ${ }^{37}$, se puede afimar que el Tratado de "Estabilidad, Coordinación y Gobernanza" y el MEDE han sido los mecanismos internacionales a los que se ha tenido que recurrir para "resolver la paradójica situación de tener una moneda común "pero no una economía común”.

\section{A) El principio de estabilidad presupuestaria y la reforma del artículo 135 de la Constitución Española}

104. Reiteradamente se ha hecho ya alusión en páginas precedentes a la grave crisis económica y financiera que España, como el resto de los países de nuestro entorno, afrontó a partir del año $2008^{38}$.

105. La situación llegó a ser tan extremadamente acuciante que el 23 de agosto de 2011 el Presidente Rodríguez Zapatero formuló una propuesta de reforma constitucional con el objetivo de contención del gasto público, iniciativa que prosperó con los apoyos del Partido Popular y Unión del Pueblo Canario.

106. Esta reforma fue el "resultado necesario de las condiciones económicas y financieras del momento y, desde luego, de las exigencias provenientes de Europa, que demandaban un equilibrio financiero y presupuestario" 39 .

107. El 27 de Septiembre de 2011 se publica en el BOE el nuevo artículo 135 de la Constitución española, entrando en vigor ese mismo día.

108. Tras esta reforma, la segunda del texto constitucional desde su promulgación en 1978, todas las Administraciones Públicas han de adecuar sus actuaciones al principio de estabilidad presupuestaria (artículo 135.1 CE), también conocida como "la regla de oro" del equilibrio presupuestario: que las previsiones presupuestarias de ingresos y de gastos se encuentren siempre en situación de equilibrio, o de superávit" ${ }^{40}$.

109. Este último precepto fue desarrollado por la Ley Orgánica 2/2012, de 27 de abril, de Estabilidad Presupuestaria y Sostenibilidad Financiera, en cuya Exposición de Motivos se afirma que "la reforma de la Constitución pretende manifestar el claro compromiso de España con las exigencias de coordinación y definición del marco de estabilidad común de la Unión Europea...siendo además España uno de los primeros países en incorporar el paquete de gobernanza económica europea a su ordenamiento jurídico interno. Además, esta Ley da cumplimiento al Tratado de "Estabilidad, Coordinación y Gobernanza" en la Unión Económica y Monetaria de 2 de marzo de 2012, garantizando una adaptación continua y automática a la normativa europea".

\footnotetext{
${ }^{36}$ Cfr. A. Aguilar Calahorro, "La Decisión Pringle en el Proceso de Constitucionalización de la Unión Europea", Op. cit.; pp.337-380.

${ }^{37}$ M. Serrano Pérez; “La Constitución Económica Europea y la Gobernanza Económica”. Revista Facultad de Derecho $y$ Ciencias Políticas, $\mathrm{n}^{\circ}$ 118, 2013, p.114.

${ }^{38}$ Cfr. J.I. García Ninet (Dir.); El Impacto de la Gran Crisis Mundial Sobre el Derecho del Trabajo y de la Seguridad Social. Su Incidencia en España, Europa y Brasil 2008-2014, Atelier. Barcelona. 2014.

${ }^{39}$ A. Bar Cendrón, "La Reforma Constitucional y la Gobernanza Económica de la Unión Europea”. Op. cit.; p.60.

${ }^{40}$ A. BAR CEndRón; La Reforma Constitucional y la Gobernanza Económica de la Unión Europea”. Op. cit.; p.61.
} 


\section{Recapitulación: La legislación de Seguridad Social, competencia nacional}

110. Se ha de partir de la base de que el régimen jurídico de las pensiones en España nunca ha sido un sistema estático sino dinámico. Sucesivas reformas se han sucedido en las últimas décadas entre las que cabría citar -siguiendo a RODRÍGUEZ INIESTA ${ }^{41}$ - las introducidas por la Ley 26/1985, 31 de julio, de Medidas Urgentes para la Racionalización de la Estructura y de la Acción Protectora de la Seguridad Social; Ley 26/1990, de 20 diciembre, por la que se establecen en la Seguridad Social prestaciones no contributivas; Ley 24/1997, de 15 de julio, de consolidación y racionalización del Sistema de Seguridad Social; Ley 28/2003, de 29 de septiembre, reguladora del Fondo de Reserva de la Seguridad Social; y Ley 40/2007, de 4 diciembre de medidas en materia de Seguridad Social.

111. Consecuentemente, la promulgación de la Ley 27/2011, de 1 agosto, de Actualización, adecuación y modernización del sistema de Seguridad Social, y de la Ley 23/2013, de 23 diciembre reguladora del Factor de Sostenibilidad e Índice de Revalorización no son sino hitos legislativos en una cadena de reformas que continúan hasta nuestros días.

112. A la vista de los Programas Nacionales de Estabilidad y Reforma presentados por España desde 2011 y las Recomendaciones específicas emanadas del Consejo de la Unión Europea para España, la conclusión que se alcanza -salvo mejor opinión- es que el Consejo se ha limitado en materia de pensiones a refrendar las reformas que España ha impulsado en el trienio 2011-2013, exactamente en los mismos términos y con el mismo alcance y extensión que son objeto de inclusión en los Planes de Reforma y en los Programas de Estabilidad.

113. No puede imputarse, por tanto, al Consejo de la Unión Europea la iniciativa de reforma concreta alguna en materia de pensiones para nuestro país.

114. Es más, a pesar de que en los últimos años el gasto en pensiones ha alcanzado records históricos y que los recursos del Fondo de Reserva han descendido en picado, no hay reformas específicas en materia de pensiones entre las recomendaciones dirigidas por el Consejo de la Unión Europea a España en los últimos cuatro años. Curiosamente, la supresión de las referencias del Consejo de la Unión Europea al tema de las pensiones en sus recomendaciones específicas anuales prácticamente coinciden en el tiempo con la pérdida de la mayoría absoluta en el Congreso del partido en el gobierno.

115. Con una cada vez mayor contestación social a la política de austeridad impuesta en años precedentes, unido a la inestabilidad política derivada del auge del nacionalismo, difícilmente habrá a corto plazo en España Gobierno alguno que pueda afrontar el desgate político que supondría cualquier nuevo intento de reforma de gran calado del sistema de pensiones a costa de reducir la cobertura y suficiencia de las mismas.

116. Igualmente, entendemos que tampoco cabe imputar a la Comisión Europea la responsabilidad en materia de reformas de pensiones, pues hay que diferenciar entre:

a. Las funciones que tiene encomendadas en el marco del Semestre Europeo.

117. Aunque es la Comisión la que elabora las recomendaciones para cada Estado miembro, éstas han de ser refrendadas por el Consejo Europeo en el mes de junio y adoptadas un mes más tarde por el Consejo de la Unión Europea.

${ }^{41}$ G. Rodríguez InIESTA, "Sinopsis de las Últimas Reformas Legislativas en el Ámbito de la Seguridad Social” en: VV.AA., El Derecho del Trabajo y la Seguridad Social en la Encrucijada, Laborum, Murcia, 2016, pp.13-29. 
118. Por tanto, resulta poco realista que los Estados vayan a aceptar recomendaciones que no hayan sido previamente "consensuadas" y estén en sintonía con los intereses políticos del partido/s en el gobierno.

119. Dicho con otras palabras, la Comisión Europea por sí misma carece de competencias para imponer a los Estados miembros ninguna reforma de su sistema de pensiones y mucho menos para legislar en dicha materia, la última palabra es siempre competencia de los Estados. Las propuestas que la Comisión Europea pueda realizar en materia de pensiones y que se materializan en una variada gama de actos (dictámenes, Libros Verdes y Libros Blancos ${ }^{42}$ ) carecen de eficacia jurídica vinculante.

120. No son fuente del Derecho de la Unión Europea conforme al artículo 288 TFUE $^{43}$.

Por tanto, no vinculan a los Estados miembros, sin perjuicio de que iniciativas concretas pudieran ser asumidas por los Gobiernos nacionales.

121. En cualquier caso y en honor a la verdad hay que reconocer que las instituciones europeas no se han erigido nunca formalmente con competencias que no les atribuyen los Tratados sino que, por el contrario, siempre han reconocido que la "UE no tiene poder para legislar sobre el diseño de los sistemas de pensiones como tales en los Estados miembros" y que cada Estado miembro es libre de determinar las particularidades de su propio régimen de seguridad social, como por ejemplo qué prestaciones se conceden, las condiciones para optar a ellas, cómo se calculan y qué cotizaciones deben abonarse ${ }^{44}$.

122. Tampoco se han cuestionado "las prerrogativas de los Estados en materia de pensiones" y se ha rechazado "que exista un sistema de pensiones ideal con un diseño válido para todos ${ }^{45}$.

123. A mayor abundamiento, la Comisión reconoce que, igual como ocurre con el empleo, las políticas de protección social y de inclusión social son responsabilidad de los Estados miembros ${ }^{46}$.

124. Tales afirmaciones reflejan la legalidad vigente tal y como ha corroborado la jurisprudencia del TJUE, que ha reconocido que el Tratado no aspira, ni aspiró en ningún momento, a crear un régimen común de protección social ${ }^{47}$ por lo que permanecen inalteradas las diferencias entre los regímenes de Seguridad de los Estados miembros ${ }^{48}$.

125. Igualmente, el Tribunal de Justicia de la Unión Europea ${ }^{49}$ ha sostenido que "las orientaciones generales de la política social definida por cada Estado miembro no pueden ser objeto de un control juris-

42 "Los Libros Blancos de la Comisión Europea son documentos que contienen propuestas de acciones de la Unión Europea en un campo específico. A veces constituyen una continuación de los Libros Verdes publicados, cuyo objetivo es iniciar un proceso de consultas a escala de la UE. El propósito de los Libros Blancos es iniciar un debate con el público, las partes interesadas, el Parlamento Europeo y el Consejo con el fin de alcanzar un consenso político".

${ }^{43}$ El Reglamento: obligatorio en todos sus elementos, es directamente aplicable en cada Estado miembro. La Directiva: obliga al Estado miembro en cuanto al resultado que deba obtenerse, exige una transposición al ordenamiento jurídico nacional y deja cierta libertad en cuanto a la forma y los medios de su aplicación. La Decisión: obligatoria en todos sus elementos, vincula a los destinatarios a quienes designa expresamente. La Recomendación y el dictamen: no obligatorios, tienen carácter de declaración.

${ }^{44}$ Comisión Europea, “Comunicación sobre revisión de la legislación de la UE en materia de coordinación”, de 13.12.2016. Disponible en: http://europa.eu/rapid/press-release_IP-16-4301_es.htm.

${ }^{45}$ Comisión Europea, Libro Verde "En Pos de unos Sistemas de Pensiones Europeos Adecuados, Sostenibles y Seguros", 2010, p.2.

${ }^{46}$ Comisión Europea, "La aportación de la UE al envejecimiento activo y la solidaridad entre generaciones", Op. Cit., p.14.

${ }^{47}$ STJCE de 21 de marzo de 1990, Cabras, 199/98, Rec. I-1023.

${ }^{48}$ STJUE de 15 de enero de 1986, Pinna, 41/84, Rec. 585. Subsisten diferencias entre los regímenes de Seguridad Social de los Estados miembros y, por consiguiente, entre los derechos de las personas que en ellos trabajan. Las diferencias de fondo y de procedimiento entre los regímenes de seguridad social de cada Estado miembro -y por ello entre los derechos de las personas que en ellos trabajan- no son afectadas por el Tratado.

${ }^{49}$ SSTJCE de 11 de junio de 1987, Teuling, 30/85, Rec.2497; de 7 de mayo de 1991, Comisión/Bélgica, 229/89, Rec. I-2205; de 19 de noviembre de 1992, Molenbroeck, 226/91, Rec. I-5963; de 17 de marzo de 1993, asuntos acumulados (Neptum), 72/91 y 73/91, Rec. 927 y de 28 de abril de 1994, Hoorn, 305/92, Rec. I-1525, entre otras. 
diccional". Hasta el punto que ni tan siquiera la normativa comunitaria resulta aplicable para determinar los requisitos de afiliación a un régimen concreto de Seguridad Social, ya sea obligatorio o voluntario ${ }^{50}$.

126. En concreto, por lo que a las pensiones se refiere, la Confederación Europea de Sindicatos hace hincapié en recordar en su respuesta al Libro Verde de la Comisión Europea, que "la Unión Europea, en virtud de los Tratados actuales, no es competente para intervenir en la organización, estructura y financiación de los sistemas obligatorios de pensiones".

127. Por último, pero no menos clarificador para comprender porqué la legislación de Seguridad Social es competencia de los Estados, hay que destacar que "frente a la Estrategia Europea de Empleo que cuenta con financiación comunitaria a través de los Fondos Estructurales, las políticas de protección social se financian con los presupuestos nacionales, sin contribución comunitaria alguna...es lógico, por tanto, que los Estados miembros no acepten ni directrices ni recomendaciones procedentes de la Comisión" ${ }^{51}$.

\section{Conclusiones}

128. Con la implantación de la moneda común los Estados de la euro zona se han vistos sometidos a una rigurosa disciplina presupuestaria, reforzada como consecuencia de la inestabilidad derivada de la situación de las finanzas públicas en los países mediterráneos tras la última -por el momento-gran crisis financiera y económica mundial que se inició en 2008.

129. En España, esta política de austeridad deriva de la imperiosa necesidad de cumplir los compromisos asumidos en materia de déficit público y nivel de deuda pública derivados del Derecho de la UE, por una parte; y de los Tratados internacionales ratificados por nuestro país, de otro lado; y cuyo incumplimiento puede acarrear graves consecuencias.

130. Ante la tesitura de tener que reducir el déficit público, y entre las múltiples opciones que el legislador nacional tenía en su haber para cumplir ese objetivo se decidió, entre otras medidas, por una paramétrica y restrictiva reforma de las pensiones de jubilaciones contributivas por ser las que mayor cuantía de gasto generan para los Presupuestos Generales del Estado.

131. Como ha reconocido la propia Comisión Europea en su Libro Blanco (2012), las reformas de jubilación en los Estados miembros se consideran un factor determinante para el buen funcionamiento de la Unión Económica y Monetaria.

132. Lo que a nuestro entender diferencia claramente las reformas en materia de pensiones en España llevadas a cabo tras la implementación del Semestre Europeo -frente a otras reformas anteriores en el tiempo- es que las del trienio 2011-2013 se enmarcan claramente dentro de una política de austeridad financiera cuyo objetivo, en última instancia, es de naturaleza eminentemente fiscal: reducir el porcentaje del PIB destinado a sufragar pensiones contributivas.

133. Pero lo cierto es que las instituciones de la Unión Europea nunca se han arrogado competencias legislativas en materia de Seguridad Social, ya que es competencia de los Estados miembros.

134. La iniciativa, contenido y alcance de la reforma del sistema de pensiones es imputable al legislador español exclusivamente. En ningún caso ordenada o impuesta por la Comisión Europea o por el Consejo de la UE.

\footnotetext{
${ }^{50}$ SSTJCE de 12 de julio de 1979, Brunori, 266/78, Rec. 2705; de 24 de abril de 1980, Coonan, 110/79, Rec.1445 y de 24 de septiembre de 1987, Rijke, 43/86, Rec. 3611.

${ }^{51}$ V. Pérez Menayo, “El Método Abierto de Coordinación en la Unión Europea: su Aplicación a las Pensiones”, Op. Cit., p.53.
} 
135. Examinados los Programas Nacionales de Estabilidad y Reforma presentados por España desde 2011 y las Recomendaciones específicas emanadas del Consejo de la Unión Europea para nuestro país se concluye que el Consejo se ha limitado en materia de pensiones a refrendar las reformas que España ha impulsado en el trienio 2011-2013, en los mismos términos y con el mismo alcance y extensión que son objeto de inclusión en los Programas de Estabilidad.

136. Asimismo puede afirmarse rotundamente que ninguno de los Tratados internacionales ratificados por España obliga o condiciona la reforma del sistema público de pensiones.

137. Podría, pues, considerarse que la reforma de las pensiones contributivas es uno de los "peajes" que ha habido que pagar en aras de la estabilidad de la moneda única (el euro).

138. Dicho con otras palabras, el fortalecimiento del Pilar Económico de la Unión Europea se ha realizado a costa de debilitar el Pilar Social.

139. Por lo que al futuro de las pensiones de jubilación se refiere no es previsible que asistamos en España a nuevas reformas paramétricas: con una cada vez mayor contestación social a la política de austeridad impuesta en años precedentes, unido a la inestabilidad política derivada del auge del nacionalismo, difícilmente habrá a corto plazo en España Gobierno alguno que pueda afrontar el desgate político que supondría cualquier nuevo intento de reforma de gran calado del sistema de pensiones a costa de reducir la cobertura y suficiencia de las mismas. 